\title{
Improving Lives in Three Dimensions: The Feasibility of 3D Printing for Creating Personalized Medical Aids in a Rural Area of Sierra Leone
}

\author{
Merel van der Stelt, ${ }^{1,2 \star}$ Arico C. Verhulst, ${ }^{2}$ Jonathan H. Vas Nunes, ${ }^{3}$ Throy A. R. Koroma, ${ }^{3}$ Wouter W. E. Nolet, ${ }^{3}$ Cornelis H. Slump, ${ }^{1}$ \\ Martin P. Grobusch, ${ }^{4}$ Thomas J. J. Maal, ${ }^{2}$ and Lars Brouwers ${ }^{5}$ \\ ${ }^{1}$ Technical Medicine, University of Twente, Enschede, The Netherlands; ${ }^{2} 3 \mathrm{D}$ Lab, Radboud University Medical Center, Nijmegen, The Netherlands; \\ ${ }^{3}$ Masanga Medical Research Unit (MMRU), Tonkolili District, Sierra Leone; ${ }^{4}$ Center for Tropical Medicine and Travel Medicine, Amsterdam \\ University Medical Centers, Amsterdam, The Netherlands; ${ }^{5}$ Department of Surgery, Radboud University Medical Center, Nijmegen,
} The Netherlands

\begin{abstract}
The aim of this feasibility study was to investigate how a 3D printer could be put to its best use in a resourcelimited healthcare setting. We have examined whether a 3D printer can contribute to making prostheses, braces, or splints for patients who underwent major limb amputation because of complex wounds, for example, due to burns and subsequent scarring, accidents, conflicts, or congenital abnormalities. During a 3-month period, we investigated the benefits of customized, 3D-printed arm prostheses, splints, and braces in Sierra Leone. Using a handheld 3D scanner and a 3D printer, patient-specific medical aids were designed, manufactured, and tested. Questionnaires regarding patient satisfaction and the functionality of the prostheses were used for a short-term follow-up. Four esthetic prostheses were designed: two prostheses of the hand, one of the forearm, and one of the entire arm. Follow-ups were conducted after 3 to 4 weeks to investigate the quality of the prostheses and to complete a patient questionnaire. Even though the prostheses primarily fulfill esthetic needs, they also exhibit some degree of functionality. In addition, four splints for hands and arms were made to prevent scar contractures after skin transplantation. Finally, a brace for a young boy with kyphoscoliosis was manufactured. The boy has accepted the brace and will be followed up in the months to come. Long-term follow-up is required to prove the sustainability of the 3D-printed brace and prosthetic arms. Further research into how to sustain and refine this project is underway.
\end{abstract}

\section{INTRODUCTION}

Sierra Leone ranks 184 of 187 on the UN development index and has a population of seven million inhabitants. ${ }^{1}$ In the aftermath of both a civil war (1991-2002) and the recent West African Ebola outbreak (2014-2016), Sierra Leone's health system is fragile, and the country is still reeling from the effects of these incidents.

Many complex wounds and amputations occur in Sierra Leone because of traffic accidents and serious infections. ${ }^{2} \mathrm{~A}$ common problem is the delayed patient presentation to the hospital, and a vast majority of patients consult traditional healers. ${ }^{3}$ Because of this combination of circumstances, an initially small medical problem often leads to irreversible damage. Furthermore, many amputees lost their limbs because of acts of violence during the civil war. ${ }^{4}$

The availability of medical aids and prostheses is still a major problem because of a lack of knowledge, low availability of materials, few trained staff, and high costs. ${ }^{5,6}$ Below-theknee prostheses typically cost around US\$100; above-theknee prostheses cost around US\$150. Prostheses for the arms are rarely made, and therefore cost estimates are not at hand. These prices are mainly high because of considerable import prices of the required materials. With an average income of US\$506 a year, buying such a prosthesis is beyond reach for most people. ${ }^{7}$ It is estimated that only $5-15 \%$ of people in countries with low income who require assistive devices and technologies have access to them. ${ }^{6}$

$3 \mathrm{D}$ printing is still a rarely used technique in resource-limited settings, especially in the healthcare sector. ${ }^{8}$ Nevertheless, we expect that communities may largely benefit from access

*Address correspondence to Merel van der Stelt, 3D Lab, Radboud University Medical Center, Geert Grooteplein Zuid 10, Nijmegen 6525 GA, The Netherlands. E-mail: merelvanderstelt@gmail.com to customized 3D-printed medical aids. Access to 3D printing, even in its most basic form, can provide a useful and essential tool for manufacturing locally relevant medical aids, such as braces, splints, and prostheses. Both simple and more complex products can be made when needed, with decreased transportation delay and lower costs.

When people are missing limbs, they often feel incomplete, which can lead to insecurity, depression, and exclusion from society. It is expected that having a prosthesis will allow them to blend in with the rest of the society and give them more confidence.

In addition to prostheses, we discovered that there is also a demand for other medical devices. Currently, there are no medical devices or surgical options for people with scoliosis in Sierra Leone. These people can be helped with a 3D-printed brace. In addition, 3D-printed splints can be made. For example, people can prevent contractures after burns or skin transplants with such splints.

In addition, we assume that esthetics plays an important role in Sierra Leone. A 3D handheld scanner enables the creation of esthetically appropriate patient-specific prostheses by using the contralateral side as a model. In this way, esthetic prostheses can be made. Finally, 3D-printed products are lightweight and, therefore, very suitable for use in prostheses and other medical aids.

We are not the first to set up a 3D laboratory in a rural area. Similar examples of the use of extrusion printers for local customized production of prosthetic arms, hands, fingers, and legs have been found in Sudan, Uganda, Pakistan, Syria, Nigeria, Madagascar, Guatemala, Nepal, Cambodia, Ecuador, and Egypt. ${ }^{9-16}$ A similar project for printing medical equipment is being set up in two hospitals in Kenya and Haiti. ${ }^{17,18} \mathrm{How}-$ ever, because of the limited research and follow-up performed on these projects, it is difficult to assess the quality of the medical aids and the extent to which the projects have been successful and are still ongoing. This shows that affordable access to this technology remains a major challenge in lowand middle-income countries. ${ }^{19}$ 


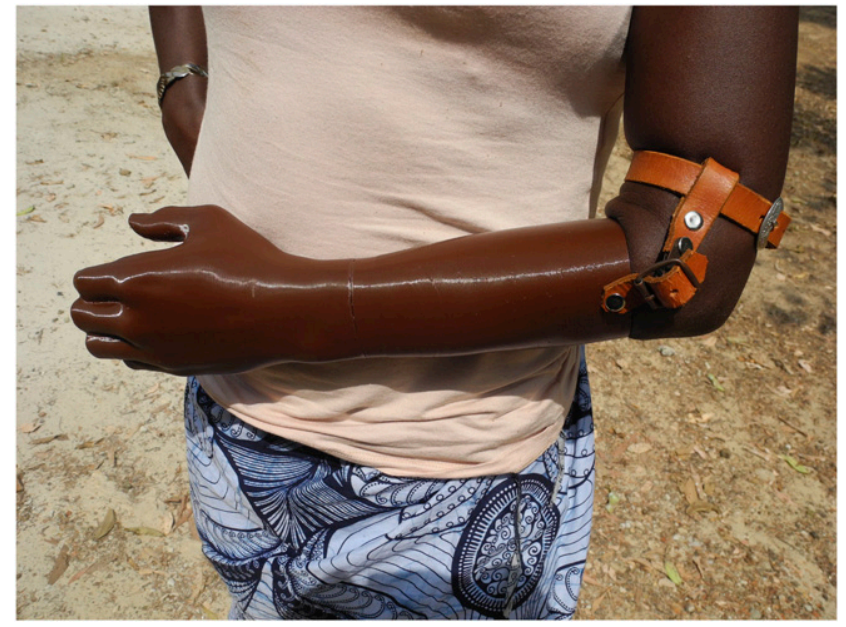

FIGURE 1. Woman with transradial amputation. She lost her arm because of a snakebite for which a traditional healer was consulted. A 3Dprinted prosthesis was made for her, with an extra belt to prevent slipping. She uses the prothesis for domestic activities, supporting her dominant hand, cooking, and esthetic purposes. (Photos with kind permission of the patient). This figure appears in color at www.ajtmh.org.

In addition, most projects that have been established make functional 3D-printed hand prostheses consisting of multiple printed parts. ${ }^{20-23}$ Although these prostheses allow a partial gripping motion, we believe them to be too fragile and complex to maintain and repair in a limited-resource setting, such as in Sierra Leone. In addition, we expect that there is more demand for a prosthesis that meets esthetic needs. With the robotic appearance of these prostheses, they seem less suitable for esthetic purposes.

The aim of this feasibility study was to investigate how a 3D printer could be put to its best use in a resource-limited healthcare setting. We examine whether a 3D printer could contribute by making prostheses, braces, and splints for patients who have undergone an amputation or who have congenital abnormalities or burns.

\section{METHODS}

During a test period of 3 months, from December 2018 until February 2019, participants were recruited from Masanga Hospital or amputation camps in the area. In Sierra Leone, a number of amputation camps, where people live together in a community, were established after the war.

Informed consent was obtained from all participants before inclusion. All participants agreed to use photos in which they wear medical aids in publications. Ethical approval for this study was obtained from the Sierra Leone Ethics and Scientific Review Committee.

Patients who required a prothesis were invited to participate in the study if the amputation occurred at least 4 months ago. Participants were not eligible when wounds on the stump were present or when the stump was painful. The wishes of the patients regarding the esthetics and functionality of the prostheses were discussed during the planning phase.

Function tests and dimensions of the stump, the unaffected limb, and other parts of the body were recorded and scanned with a handheld 3D scanner (Einscanner Pro, Shining 3D Technology, Hangzhou, China). ${ }^{24}$ Subsequently, a design was made in Meshmixer (Autodesk Meshmixer 3.5, Toronto,

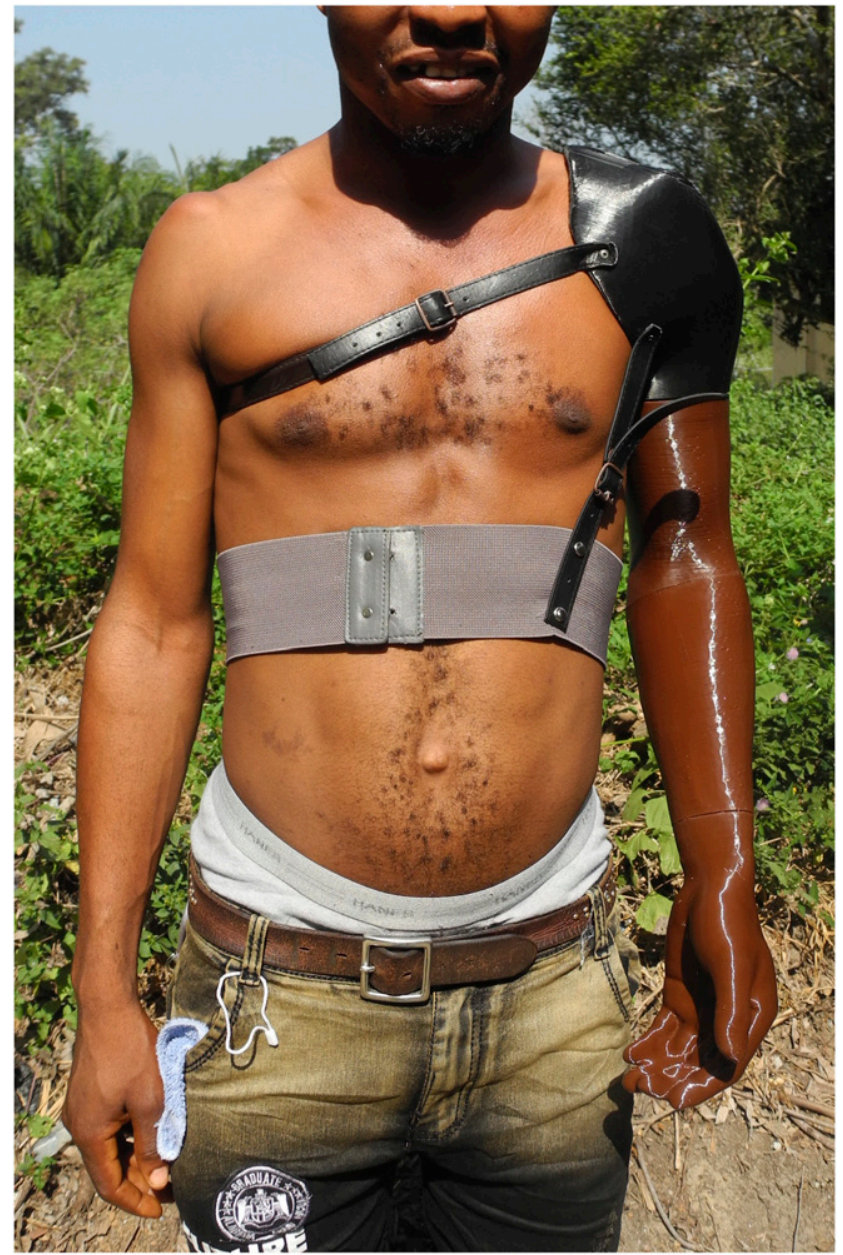

FIGURE 2. Man with shoulder disarticulation. He lost his arm because of a fall accident causing a fracture, after which a traditional healer was consulted. The arm became infected, resulting in necrosis. A 3D-printed prosthesis was made for him, with a belt to attach the prosthesis to his body. He uses the prosthesis for esthetic purposes, especially when he goes to school. (Photos with kind permission of the patient). This figure appears in color at www.ajtmh.org.

Canada). ${ }^{25}$ For the prostheses, a custom-made socket was designed with a mirrored version of the contralateral biological arm. Using the 3D printer (Ultimaker 2 + Ultimaker BV, Geldermalsen, the Netherlands) located at the Masanga Hospital, medical aids can be manufactured quickly. The printer uses a fused deposition modeling technique. This is a technique in which the print head extrudes melted thermoplastic filaments in a layering process to create a 3D object. Polylactic acid (PLA) (Ultimaker BV) filaments were used to create the medical aids. ${ }^{26}$ Uninterruptible power supply was used to supply voltage to the printer to prevent power changes and outages.

Finally, additional adjustments were made together with the local prosthetics specialist. When the prosthesis or other medical aid was finished, the participant was instructed to return to the hospital to fit the prosthesis and test its functionality. Minor adjustments were made according to need, using leather straps for belts and foam to prevent pressure spots. If the prosthesis was considered satisfactory by both the patient and healthcare worker, the patient was allowed to take it home. A Dutch prosthetic specialist was involved via teleconferencing for any questions and advice. 
TABLE 1

Summary of completed questionnaires by patients who had obtained 3D-printed prostheses

Cause of amputation

Number of hours of daily use of the prosthesis:

Does the prosthesis give you more confidence? What would you prefer: esthetic or functional prosthesis? Purpose of use

Complaints related to prosthesis (stump pain, skin problems, phantom pain, and suspension)

\author{
1/4: Civil War \\ 1/4: snake bite and traditional healing \\ $1 / 4$ : fall accident and traditional healing \\ 1/4: burns \\ 3/4: more than 6 hours \\ 1/4: 4 hours \\ 4/4: yes \\ 4/4: esthetics is just as important as function \\ Case 1: grabbing things, supporting the dominant hand, \\ getting dressed, cooking, and aesthetic purposes \\ Case 2: domestic activities, supporting the dominant hand, \\ cooking, and esthetic purposes \\ Case 3: esthetic purposes \\ Case 4: esthetic purposes \\ 4/4: No
}

Patients who received a prosthesis were asked to complete questionnaires to assess their satisfaction and the functionality of the prosthesis after 3-4 weeks. This questionnaire was based on an existing questionnaire made for upper limb prostheses but adapted to an African situation with additional questions (Supplemental Appendix B). ${ }^{27}$

\section{RESULTS}

Four esthetic prostheses were made: two prostheses of the hand, one of the forearm, and one of the total arm (Figures 1 and 2). Even though the prostheses are primarily esthetic, they also exhibit some degree of functionality. For example, the prosthetic arm can provide support for the contralateral biological arm, and participants seem to have more self-confidence when wearing the prosthesis. Table 1 provides information obtained from the questionnaire. All patients were still wearing the prosthesis after 3-4 weeks of follow-up. Three of the four patients were using the prosthesis for more than 6 hours a day. One patient used the prosthesis for 4 hours a day. In addition, all four patients indicated that they considered esthetics just as important as functionality and that the prosthesis provided extra self-confidence. No complications occurred in the short term.

Four splints for hands and arms were made to prevent scar contractures after skin transplantation (Figure 3). The splints were well worn by the time the patients recovered in the hospital, and after discharge, no follow-up was possible.

In addition, a noncorrecting brace for a 7-year-old boy with kyphoscoliosis was made (Figure 4). We hope that the brace will provide enough support to prevent a deterioration in the kyphoscoliosis. After 15 weeks, he was still wearing the brace, and it did not cause any pressure spots or skin irritation. In the future, he will be followed up with to explore the possibility of replacing the braces as he continues to grow.

The medical aids are lightweight. A forearm prosthesis weighs approximately $300 \mathrm{~g}$ (\$14 USD in material costs), and the brace weights approximately $340 \mathrm{~g}$ (\$16 USD in material costs). This ensures that the medical aids can easily be worn by children.

\section{DISCUSSION}

In this study, we investigated how a 3D printer can be put to best use in a resource-limited healthcare setting. In the 3-month pilot phase period from December 2018 to February 2019, we examined whether a 3D printer could contribute by making prostheses, braces, and splints for patients who have undergone an amputation or have congenital abnormalities or burns. Four esthetic prostheses were made: two prostheses of the hand, one of the forearm, and one of the total arm. In
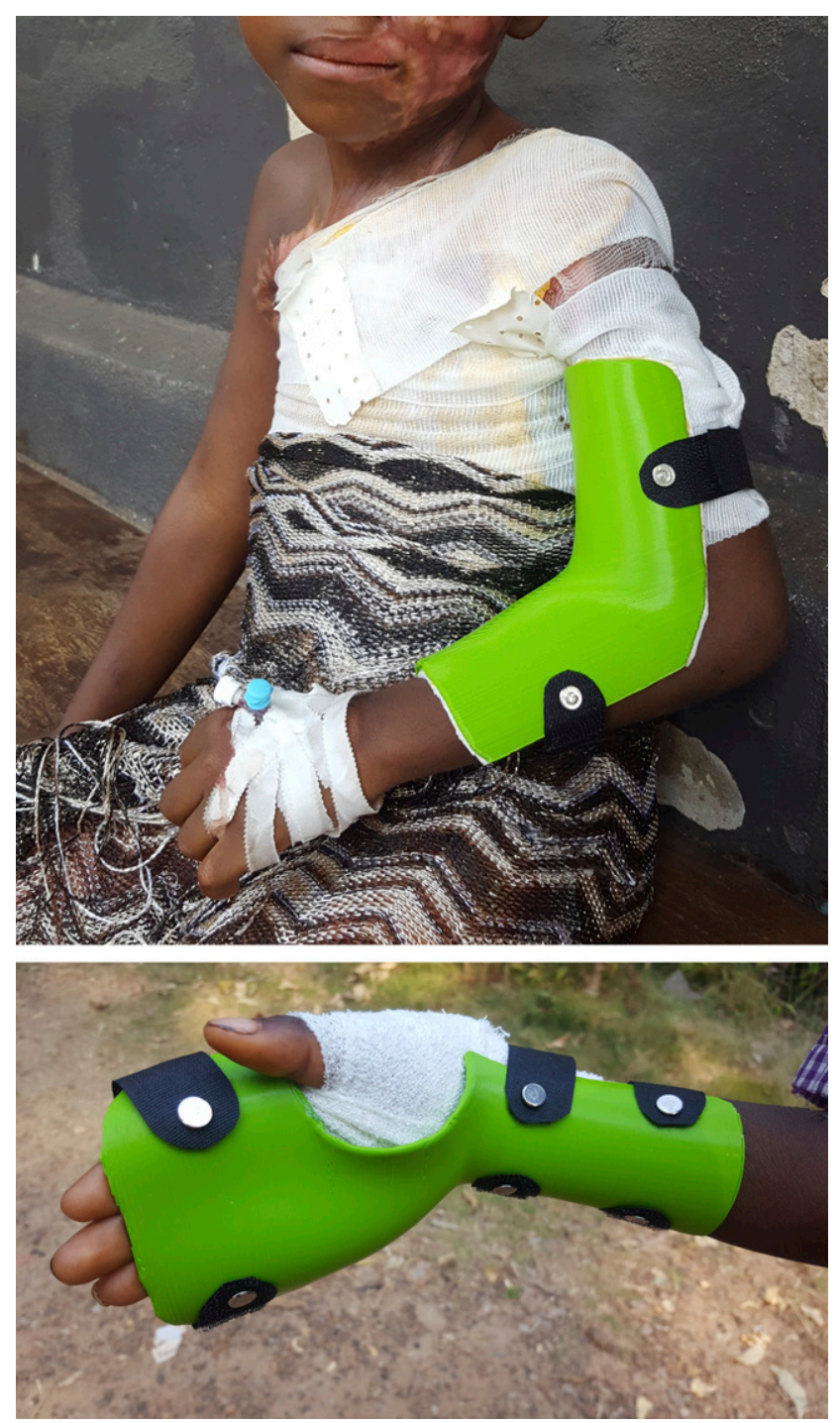

FIGURE 3. 3D-printed splints for hands and arms to prevent burn scar contractures after skin transplantation. This figure appears in color at www.ajtmh.org. 

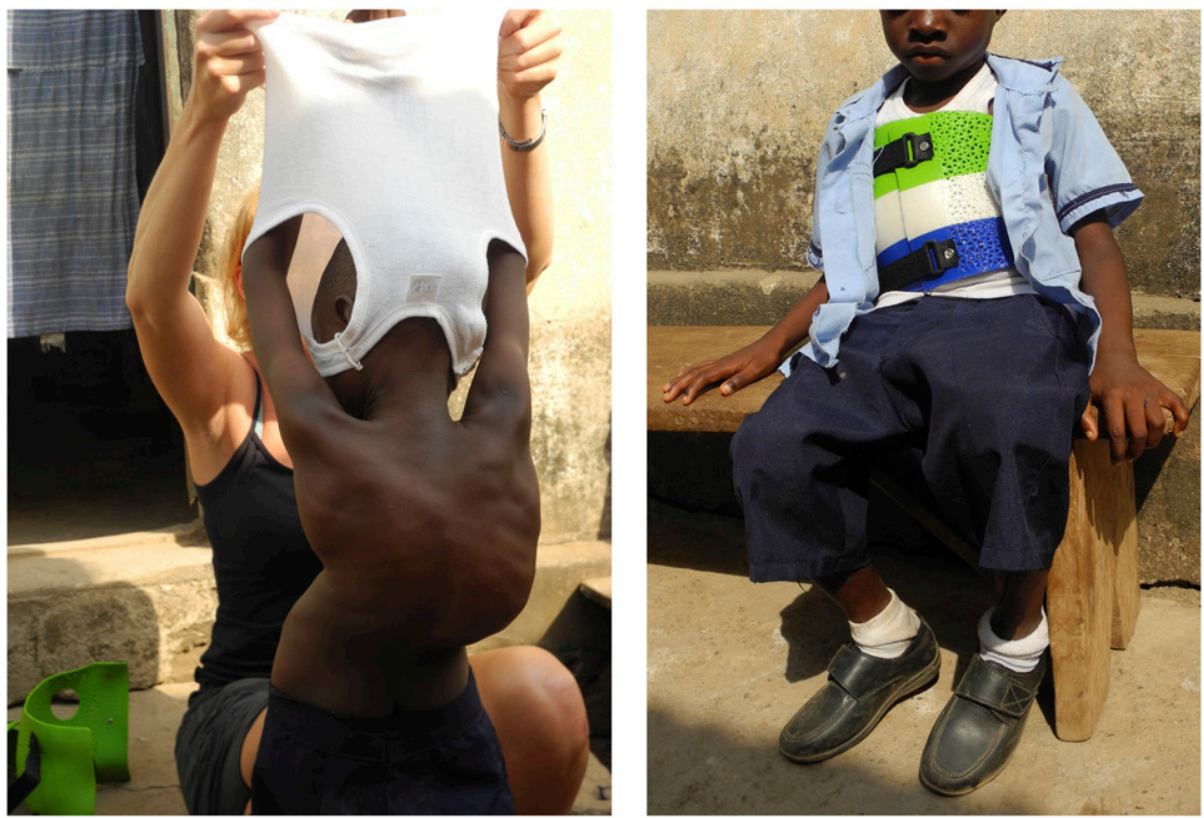

FIGURE 4. 3D-printed non-correcting brace for a 7-year-old boy with kyphoscoliosis. (Photos with kind permission of the parents). This figure appears in color at www.ajtmh.org.

addition to the esthetic function, which appears to play a major role in Sierra Leone, the prostheses also seem to exhibit some degree of functionality. Moreover, four splints for hands and arms were made to prevent burn scar contractures after skin transplantation. Finally, a brace for a 7-yearold boy with kyphoscoliosis was made. 3D-printing materials are lightweight and therefore suitable for making medical aids. In addition, material costs for a prosthesis of the forearm are only approximately \$14 USD, and for a brace, \$16 USD. These are costs that someone from Sierra Leone could afford.

It has been found that esthetics plays an important role in Sierra Leone. All participants indicated that esthetics was just as important as functionality. Wearing a prosthesis gave them more self-confidence, allowing them to increasingly participate in society. The patient with the shoulder disarticulation even felt confident enough to go to the university again. The woman with the wrist disarticulation always carried her hand under her skirt, which denied her the functionality of the entire arm. After fitting an esthetic prosthesis, she uses her arm again. The woman with the amputated fingers always carried a bag around her hand, which completely disabled the functionality of her hand. Her new prosthesis makes her feel that she has a normal hand again; her regained confidence is encouraging her to participate more in the community again. These examples indicate that 3Dprinted esthetic prostheses can be of great added value in restoring confidence. This increases the usage of the amputated $\operatorname{limb}$ and the prosthesis. Because the prosthesis was made in a gripping position, the hand can be used as a hook to pick things up and can serve as support for the dominant hand. This shows that, in addition to its esthetic function, prostheses also restore part of the functionality of a limb.

This study also has limitations. Our study population was small, and only short-term follow-up was performed at the time of the writing of this article. In the future, we hope to include more patients and perform a longer follow-up. During follow-up, we noticed that it was sometimes difficult for participants to complete the questionnaires. Many people had no formal or higher education, which led to questions being misinterpreted easily, making the results less reliable. Questionnaires must be kept as short and simple as possible. For subsequent projects, we want to prepare an extensive questionnaire that includes a quality of life test. By providing a questionnaire before and after receiving the prosthesis, the questionnaire scores could be compared with each other, and the added value of the prosthesis can be measured.

The prostheses are made from PLA, which is one of the least expensive printing materials. The present material costs of a functional 3D-printed transitional hand prosthesis are approximately $\$ 50$ USD. ${ }^{21}$ We have shown that a simple esthetic prosthesis, consisting of fewer types of material and fewer parts, can reduce the costs even more. Material costs for a prosthesis of the forearm are approximately \$14 USD. However, PLA is probably not the most suitable material because it absorbs moisture, can deform at high temperatures, and is not completely resistant to UV radiation. We have not seen any difficulties with this in the short term. Longer term assessments will indicate whether PLA is suitable for the circumstances of Sierra Leone.

For a project such as this to become independent from outside, it must be made sustainable. This means that the prostheses must be affordable for the local population and that the project can be carried out by the local population itself. This is a major challenge due to the limited knowledge available in Sierra Leone in the field of 3D printing. In the longer term, we hope to be able to train local collaborators to run the project independently. To achieve this, the process of making the prostheses must be made as standardized and simple as possible. In the current procedure, a design was made in a freely available design program MeshMixer. To learn how to work with this program, certain knowledge of computers and design is needed, which requires substantial time and effort to learn. We hope to write a software program to automate this process so that these time-consuming and difficult steps can be skipped, making the process of learning to design a 
prothesis easier. Long-term follow-up needs to be performed to prove the sustainability of the 3D-printed brace and prosthetic arms. We hope to be able to visit the participants again after 6 months to investigate their use of the prostheses.

This study has proven the added value of 3D-printed medical aids in low-income countries such as Sierra Leone. In the coming years, we hope to further expand the 3D laboratory so that we can make more arm prostheses and other medial aids and improve them. Finally, we have seen that there is also a great demand for affordable leg prostheses. Although these prostheses must meet a wider range of requirements, we hope to investigate whether 3D printing can also be of value in this area.

\section{CONCLUSION}

We believe that because of its low cost and relatively easyto-use technology, 3D printing is useful for healthcare purposes, especially in resource-limited settings. The first prosthetic results seem to be positive. Even though the prostheses are primarily esthetic, they also exhibit some degree of functionality. Long-term follow-up needs to be performed to prove the sustainability of the 3D-printed brace and prosthetic arms. Training local staff to pursue follow-up data collection and future engagement to safeguard long-term funding will be the next steps toward achieving sustainability.

Received May 9, 2019. Accepted for publication November 20, 2019.

Published online February 24, 2020.

Note: Supplemental Appendix appears at www.ajtmh.org.

Acknowledgments: The 3D printer was donated by Ultimaker (Ultimaker BV, Geldermalsen). In addition, we were allowed to borrow the handheld 3D scanner (Einscanner Pro) from Makerpoint (Makerpoint BV, Arnhem). To both companies, we express our gratitude.

Financial support: Internship allowance of 2,500 euros, obtained from the University of Twente for buying materials, ethical approval, and transport.

Authors' addresses: Merel van der Stelt and Cornelis H. Slump, Technical Medicine, University of Twente, Enschede, The Netherlands, E-mails: merelvanderstelt@gmail.com and c.h.slump@ utwente.nl. Arico C. Verhulst and Thomas J. J. Maal, 3D Lab, Radboud University Medical Center, Nijmegen, The Netherlands, E-mails: arico.verhulst@radboudumc.nl and thomas.maal@ radboudumc.nl. Jonathan $\mathrm{H}$. Vas Nunes, Throy A. R. Koroma, and Wouter W. E. Nolet, Masanga Medical Research Unit (MMRU), Tonkolili District, Sierra Leone, E-mails: jhvasnunes@gmail.com and wouternolet@gmail.com. Martin P. Grobusch, Center for Tropical Medicine and Travel Medicine, Amsterdam University Medical Centers, Amsterdam, The Netherlands, E-mail: m.p.grobusch@ amsterdamumc.uva.nl. Lars Brouwers, Department of Surgery, Radboud University Medical Center, Nijmegen, The Netherlands, E-mail: I.brouwers@etz.nl.

\section{REFERENCES}

1. United Nations Development Programme, 2018. Human Development Indices and Indicators 2018. New York, NY: The United Nations Development Programme.

2. Stewart KA, Groen RS, Kamara TB, Farahzad MM, Samai M, Cassidy LD, Kushner AL, Wren SM, 2013. Traumatic injuries in developing countries: report from a nationwide cross-sectional survey of Sierra Leone. JAMA Surg 148: 463-469.

3. Wong EG, Groen RS, Kamara TB, Stewart KA, Cassidy LD, Samai M, Kushner AL, Wren SM, 2014. Burns in Sierra Leone: a population-based assessment. Burns 40: 1748-1753.

4. Crompton J, Kingham TP, Kamara TB, Brennan MF, Kushner AL, 2010. Comparison of surgical care deficiencies between US civil war hospitals and present-day hospitals in Sierra Leone, World J Surg 34: 1743-1747.

5. AHO, 2019. Uganda: Medical Products, Vaccines, Infrastructures and Equipment-AHO. Available at: http://www.aho.afro.who.int/ profiles_information/index.php/Uganda:Medical_products, vaccines,_infrastructures_and_equipment. Accessed May 6 , 2019.

6. Matter R, Harniss M, Oderud T, Borg J, Eide AH, 2017. Assistive technology in resource-limited environments: a scoping review. Disabil Rehabil Assist Technol 12: 105-114.

7. World Bank, 2016. Sierra Leone Overview. The World Bank. Available at: http://www.worldbank.org/en/country/sierraleone/ overview. Accessed May 06, 2019.

8. Schonwetter T, Van Wiele B, 2018. 3D Printing: Enabler of Social Entrepreneurship in Africa? the Roles of FabLabs and LowCost 3D Printers. Cape Town, South Africa: Open African Innovation Research (Open AIR).

9. Banks K, Brown L, 2014. Project Daniel: 3D Printing Prosthetic Arms for Children in Sudan - National Geographic Society Newsroom, Available at: https://blog.nationalgeographic.org/ 2014/10/06/project-daniel-3d-printing-prosthetic-arms-forchildren-in-sudan/. Accessed March 06, 2019.

10. Nia Technologies, 2016. Nia Technologies|Nia Technologies. Available at: https://niatech.org/. Accessed March 06, 2019.

11. Bender K, Chartoff H, Hoppe M, 2014. Printing Prosthetic Solutions. 9548. Worcester, MA: Worcester Polytechnic Institute (WPI).

12. Medecins Sans Frontieres, 2016. Innovation |3D Printed Prosthetic Limbs for Refugees. London, United Kingdom: MSF. Available at: https://www.msf.org.uk/video/innovation-3d-printedprosthetic-limbs-refugees. Accessed March 06, 2019.

13. Enabling The Future, 2015. E-NABLING Africa 3D Printed Hands in Nigeria. Enabling The Future. Available at: http://enablingthefuture. org/2015/10/24/e-nabling-africa-•-3d-printed-hands-in-nigeria/. Accessed March 06, 2019.

14. Tobor N, 2018. Trial of 3D Printing Prosthetic Limbs in Madagascar and Togo. Available at: https://www.iafrikan.com/2018/07/04/ togo-hi-tech-orthopaedic-care/. Accessed March 06, 2019.

15. Kennett O, 2015. 3D Printing Prosthetic Limbs, Available at: https://www.legs4africa.org/3d-printing-prosthetic-legs/. Accessed March 06, 2019.

16. Dechev N, 2019. Victoria-Hand1 Available at: https://www. victoriahandproject.com/. Accessed May 06, 2019.

17. Kozminski N, 2017. Kijenzi - Humanitarian 3D Printing in the US and Kenya. Available at: https://medtechkijenzi.wordpress.com/. Accessed March 04, 2019.

18. Hostettler S, Besson SN, Bolay J-C, 2015. Hostettler S, Hazboun E, Bolay J-C, eds. Technologies for Development: What is Essential? Cham Switzerland: Springer International Publishing AG.

19. Diment LE, Thompson MS, Bergmann JHM, 2018. Three-dimensional printed upper-limb prostheses lack randomised controlled trials: a systematic review. Prosthet Orthot Int 42: 7-13.

20. Zuniga JM, Peck J, Srivastava R, Katsavelis D, Carson A, 2016. An open source 3D-printed transitional hand prosthesis for children. J Prosthetics Orthot 28: 103-108.

21. Zuniga J, Katsavelis D, Peck J, Stollberg J, Petrykowski M, Carson A, Fernandez C, 2015. Cyborg beast: a low-cost 3dprinted prosthetic hand for children with upper-limb differences. BMC Res Notes 8: 10.

22. Tanaka KS, Lightdale-Miric N, 2016. Advances in 3D-printed pediatric prostheses for upper extremity differences. J Bone Joint Surg Am 98: 1320-1326.

23. Xu G, Gao L, Tao K, Wan S, Lin Y, Xiong A, Kang B, Zeng H, 2017. Three-dimensional-printed upper limb prosthesis for a child with traumatic amputation of right wrist. Medicine (Baltimore) 96: 52.

24. Hagzhou Shining 3D Tech Co., Ltd, 2018. EinScan-Pro Guide Book. Available at: https://www.einscan.com/. Accessed October 28, 2019.

25. Autodesk, 2017. Autodesk Meshmixer version 3.5.474. San Rafael, CA. (Software).

26. Ultimaker, 2018. Ultimaker Tough PLA Material: Create Durable Prototypes and Tooling. Available at: https://ultimaker.com/ materials/tough-pla. Accessed October 09, 2019.

27. Gaber TA, Gardner CM, Kirker SG, 2001. Silicone roll-on suspension for upper limb prostheses: users' views. Prosthet Orthot Int 25: 113-118. 